\title{
Expectant Management of Severe COVID-19 Pneumonia in Late Preterm Pregnancy and Subsequent Cholecystitis: Lessons Learned
}

\author{
Neggin B. Mokhtari, MD ${ }^{1}$ Daphnie Drassinower, MD ${ }^{2}$ Lindsey A. Orr, MD ${ }^{3}$ Nathan K. Cobb, MD \\ Oscar L. Mims Jr., MD² Helain J. Landy, MD² \\ ${ }^{1}$ Department of Obstetrics and Gynecology, MedStar Washington \\ Hospital Center/Georgetown University, Washington, \\ District of Columbia \\ 2 Division of Maternal Fetal Medicine, MedStar Georgetown \\ University, Washington, District of Columbia \\ ${ }^{3}$ Division of Pulmonary and Critical Care Medicine, MedStar \\ Georgetown University, Washington, District of Columbia \\ Address for correspondence Neggin B. Mokhtari, MD, 110 Irving \\ Street, NW Office 5B-39, Washington, DC 20010 \\ (e-mail: Neggin.mokhtari@medstar.net). \\ Am J Perinatol Rep 2021;11:e29-e33.
}

\begin{abstract}
Keywords

- coronavirus

- COVID-19

- pregnancy

- cholecystitis

- early term gestation
\end{abstract}

Introduction Since the emergence of coronavirus disease 2019 (COVID-19) as a pandemic in March 2020, research and guidance have been published with regard to the management of infection and considerations in pregnancy, but much is still unknown. Pregnant women with COVID-19 infection are more likely to be hospitalized and are at increased risk for intensive care unit admissions and intubation than nonpregnant women with COVID-19 infection. The optimal timing of delivery among pregnant women with COVID-19 infection has not been established at this time, especially when the infection arises in late preterm and early term gestation. It is suggested that COVID-19 infection should not be considered a sole indication for delivery. The risks and benefits of prolonging pregnancy versus delivery should be taken into consideration at any given gestational age in a patient with COVID-19 infection.

Case Report We report a case of a patient in the late third trimester of pregnancy that presented with severe COVID-19 infection and was managed expectantly through her disease course with improvement of respiratory status without necessitating delivery. We also discuss the unique development of cholecystitis in her hospitalization that may represent another clinical association to COVID-19 infection.

Conclusion This case illustrates that delaying delivery is an option even in later gestational ages for maternal stabilization. A multidisciplinary approach and teamwork is needed to manage pregnant women with COVID-19 infection for optimal outcomes for both mother and fetus. received

July 29, 2020

accepted after revision

September 24, 2020
DOI https://doi.org/

$10.1055 / \mathrm{s}-0040-1721672$. ISSN 2157-6998.

\footnotetext{
(C) 2021. The Author(s).

This is an open access article published by Thieme under the terms of the Creative Commons Attribution-NonDerivative-NonCommercial-License, permitting copying and reproduction so long as the original work is given appropriate credit. Contents may not be used for commercial purposes, or adapted, remixed, transformed or built upon. (https://creativecommons.org/ licenses/by-nc-nd/4.0/)

Thieme Medical Publishers, Inc., 333 Seventh Avenue, 18th Floor, New York, NY 10001, USA
} 


\section{Key Points}

- Delaying delivery in severe coronavirus disease 2019 (COVID-19) infection is a reasonable option even in late gestation.

- A multidisciplinary team is of utmost importance when managing a pregnant woman with COVID-19.

- Other clinical sequalae such as cholecystitis may arise in the setting of COVID-19 infection.

Timing and mode of delivery in patients with severe COVID-19 pneumonia have not been established and making these decisions is left to the discretion of a multidisciplinary team usually involving maternal-fetal medicine specialists, obstetricians, neonatologists, intensivists, anesthesiologists, infectious disease specialists, and internists. In pregnancy, the enlarging uterus results in an upward displacement of the diaphragm which leads to a decreased functional reserve capacity and expiratory reserve volume. ${ }^{1}$ Improvement in lung mechanics and oxygenation by early delivery is theoretical and has not yet been shown to be beneficial in large case series or studies. ${ }^{2-4}$ Given that there is little knowledge about the ideal timing of delivery in a patient with severe COVID-19 pneumonia, multidisciplinary teams must consider the risks and benefits of delivery to determine the optimal timing considering risks and benefits for the mother and the fetus. ${ }^{4}$

Additionally, COVID-19 infection has been shown to affect multiple organ systems including the lung, heart, liver, and kidney, likely due to the expression of the angiotensinconverting enzyme (ACE)-2 receptor. ${ }^{5-8}$ No reports to date have demonstrated COVID-19 infection to the gallbladder, but given the presence of the ACE2 receptor, it is possible that it may lead to complications in the organ. ${ }^{5}$

We report a case of a woman in the late third trimester of pregnancy that presented with severe COVID-19 infection, developed cholecystitis, and was managed expectantly through her disease course without necessitating delivery. We present this patient case to highlight the following important issues-presence of false negative testing with rapid COVID-19 tests; multidisciplinary approach to management of a pregnant COVID-19 patient; the potential benefit of delaying delivery in the setting of severe COVID-19 infection for maternal stabilization without fetal harm; and possible association of cholecystitis in the context of COVID-19 infection.

\section{Case Report}

We present the case of a 16-year-old primigravida at 35 weeks and 1 day gestation that was transferred to a tertiary care center in the setting of acute hypoxemic respiratory failure from COVID-19 pneumonia. Her past medical history was only significant for depression with history of self-harm (cutting). She presented to the outside hospital with a 2-day history of fever, myalgias, shortness of breath, and dry cough. She had no labor complaints. She had been seen in the Emergency Department of the same institution the previous day, diagnosed with pneumonia, and discharged home with antibiotics, but returned when symptoms worsened. On second presentation, she was tachypneic with 40 to 60 breaths/min and oxygen desaturation to less than $90 \%$ on room air. She required high flow nasal cannula with oxygen at $20 \mathrm{~L} / \mathrm{min}$ with $50 \%$ fraction of inspired oxygen $\left(\mathrm{FiO}_{2}\right)$. Rapid severe acute respiratory syndrome coronavirus 2 (SARS-CoV-2) testing was negative through the Abbott ID NOW COVID-19 SARS-CoV-2 assay. A chest radiograph revealed bilateral infiltrates with a dense consolidation in the right lower lobe (-Fig. 1). Given high suspicion of COVID19 infection, polymerase chain reaction (PCR) testing was sent and was positive. She was transferred to our medical intensive care unit (MICU) for a higher level of care.

The patient had not sought prenatal care. In the MICU, bedside obstetric ultrasound confirmed dating by her sure last menstrual period. A singleton fetus was seen in the cephalic presentation with normal limited anatomy, a posterior placenta, and estimated fetal weight of $2,473 \mathrm{~g}$; amniotic fluid index was normal and fetal movements were seen. Nonstress test was reactive, Category 1 , and biophysical profile was $10 / 10$. She was managed by adult critical care with consultation from the anesthesiology, maternal fetal medicine, and infectious disease services. Initial laboratories were notable for mild anemia (hemoglobin $10.0 \mathrm{~g} / \mathrm{dL}$ ), low lymphocyte count $(1.0 \mathrm{k} / \mathrm{uL})$, elevated C-reactive protein $(129 \mathrm{mg} / \mathrm{L})$, elevated Ddimer $(3.47 \mu \mathrm{g} / \mathrm{mL})$, with normal ferritin, aspartate aminotransferase (AST), alanine aminotransferase (ALT), and interleukin-6 levels. - Table 1 illustrates trend of laboratories throughout her hospitalization. Azithromycin and ceftriaxone were administered for pneumonia treatment, as well as scheduled bronchodilator therapy. Prophylactic anticoagulation with subcutaneous heparin was initiated and maintained throughout her hospitalization. Late preterm antenatal steroids were not administered.

In the first 24 hours she required a maximum of $30 \mathrm{~L} / \mathrm{min}$ of high flow nasal cannula at $50 \% \mathrm{FiO}_{2}$ with a respiratory rate of 40 to 50 breaths/min and there was a concern that she would require intubation. Planned delivery was considered to potentially improve her respiratory condition and several multidisciplinary meetings were held. We agreed to monitor her and the fetal conditions closely and outlined a plan for delivery if her respiratory status worsened. Over the next several days, she practiced frequent self-proning and rotation to the lateral decubitus positions and used frequent incentive spirometry. Arterial blood gases revealed improved oxygenation on high flow nasal cannula. Fetal monitoring was reassuring three times a day and there were no signs of preterm labor. Her respiratory status improved over the next 2 days, no longer requiring supplemental oxygen but tachycardia and tachypneic persisted with limited activity. Within that 48-hour period, C-reactive protein levels returned to normal, but D-dimers remained elevated. On hospital day 6, the patient developed nausea and vomiting with elevated AST and ALT with values of 49 and 48 $\mathrm{u} / \mathrm{L}$, respectively. Right upper quadrant ultrasound revealed 


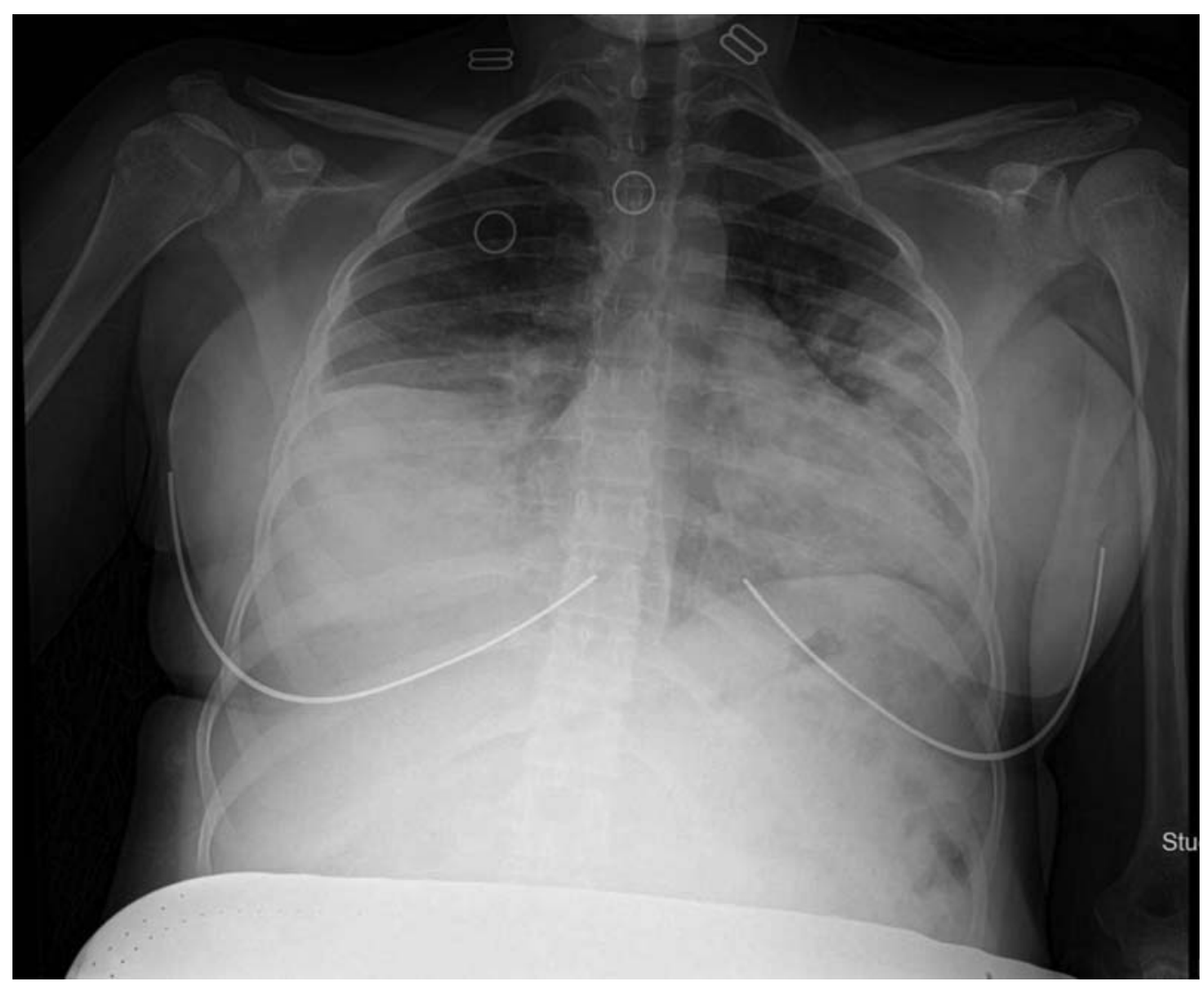

Fig. 1 Chest radiograph. Infiltrates in bilateral lung fields, with worse dense opacification in the right lower lung, with air bronchogram, compatible with multilobar pneumonia.

biliary sludge and numerous stones within the gall bladder with a positive Murphy's sign, suggesting acute cholecystitis. The general surgery team was consulted and conservative management with IV antibiotics was recommended; her condition continued to improve daily. She was discharged home on hospital day 13 with a plan for weekly follow-up.

The patient did not present for scheduled follow-up appointments despite multiple attempts to contact her. She presented to an outside hospital at 40 weeks and 2 days with contractions and progressed in labor without complications. She had an uncomplicated spontaneous vaginal delivery of a live female infant weighing $3,010 \mathrm{~g}$ with Apgar scores of 8 and 9 at 1 and 5 minutes, respectively. The estimated blood loss was $200 \mathrm{~mL}$ and she was discharged home on postpartum day 2 without complications. The infant tested negative for COVID-19 infection. A follow-up phone call confirmed that the patient was doing well at home without any further medical concerns.

\section{Discussion}

We report a case of severe COVID-19 pneumonia in the late third trimester that was expectantly managed and improved without delivery, and possibly the first case of cholecystitis associated with COVID-19 infection.

The patient had a negative result using the Abbott ID NOW COVID-19 SARS-CoV-2 assay. Given high suspicion for COVID-19, a real time-PCR was performed and was positive.
Abbott laboratories state that the shortest turnaround time for their ID NOW COVID-19 rapid test is 13 minutes, referencing multiple studies in their press release which cite sensitivity ranging from 91.3 to $100 \%$, and specificity ranging from 98.6 to $100 \%$ when compared with Roche cobas SARS-CoV-2 assay or other laboratory-based PCR assays. ${ }^{9}$ When comparing Abbott ID NOW to the Cepheid Xpert Xpress SARS-CoV-2 platform, the negative percentage agreement was $98.5 \%$, suggesting false positive rates from the ID NOW assay are very low, however, false negative rates have been reported with increasing frequency and raise concern as a stand-alone test. ${ }^{10}$

The patient met criteria for severe COVID-19 disease based on a respiratory rate greater than 30 beats per minute (bpm) and hypoxia with oxygen saturation of less than or equal to 93\%. ${ }^{4}$ Other criteria for severe COVID-19 disease include a ratio of arterial partial pressure of oxygen to fraction of inspired oxygen of less than 300 and/or greater than $50 \%$ of lung involvement on imaging. 4

COVID-19 itself is not an indication for delivery; our multidisciplinary discussions included the argument that there may be little benefit to prolonging a pregnancy in the setting of such severe disease. ${ }^{2,4}$ Although late preterm infants account for most of the admissions to the neonatal intensive care units, and are more likely to have long-term neurodevelopmental problems, respiratory complications, and infant death than those born at term, the overall neonatal outcome is expected to be favorable. ${ }^{11}$ Expectant management for fetal benefit was not felt to justify any maternal risk. In fact, a case series noted maternal 
Table 1 Laboratory values and trends through hospitalization

\begin{tabular}{|c|c|c|c|c|}
\hline & Hospital day 1 & Hospital day 4 & Hospital day 7 & Hospital day 13 \\
\hline White blood cell (k/uL) & 7.2 & 5.2 & 6.8 & 7.1 \\
\hline Hemoglobin (g/dL) & $10.0^{\mathrm{b}}$ & $10.1^{\mathrm{b}}$ & $10.6^{\mathrm{b}}$ & 10.8 \\
\hline Hematocrit (\%) & $32.3^{b}$ & $31.2^{\mathrm{b}}$ & $32.0^{b}$ & 33.5 \\
\hline Platelet (K/uL) & 236 & 316 & $410^{\mathrm{a}}$ & $372^{\mathrm{a}}$ \\
\hline Absolute lymphocyte (k/uL) & $1.0^{\mathrm{b}}$ & 1.8 & 1.9 & 2.0 \\
\hline Erythrocyte sedimentation rate $(\mathrm{mm} / \mathrm{h})$ & $66^{\mathrm{a}}$ & $72^{\mathrm{a}}$ & c & c \\
\hline C-reactive protein $(\mathrm{mg} / \mathrm{L})$ & $129^{a}$ & $13.3^{\mathrm{a}}$ & $\mathrm{c}$ & c \\
\hline Interleukin-6 (pg/mL) & $<5$ & c & c & c \\
\hline Ferritin $(\mathrm{ng} / \mathrm{mL})$ & 60 & 47.3 & $\bar{c}$ & c \\
\hline Sodium (mmol/L) & 142 & 142 & 140 & 140 \\
\hline Potassium (mmol/L) & 4.3 & 3.8 & 3.9 & 4.0 \\
\hline Chloride (mmol/L) & $111^{\mathrm{a}}$ & $111^{\mathrm{a}}$ & $108^{a}$ & 107 \\
\hline Creatinine $(\mathrm{mg} / \mathrm{dL})$ & 0.60 & $0.50^{\mathrm{b}}$ & $0.41^{\mathrm{b}}$ & $0.39^{\mathrm{b}}$ \\
\hline Glucose (mg/dL) & 76 & 80 & 82 & 75 \\
\hline Aspartate amniotransferase (units/L) & 21 & 31 & $49^{a}$ & 45 \\
\hline Alanine amniotransferase (units/L) & $12^{\mathrm{b}}$ & 16 & $48^{\mathrm{a}}$ & $48^{\mathrm{a}}$ \\
\hline Creatinine kinase (units/L) & $11^{\mathrm{b}}$ & $16^{\mathrm{b}}$ & c & c \\
\hline Lactate dehydrogenase (units/L) & 194 & 187 & c & c \\
\hline Fibrinogen $(\mathrm{mg} / \mathrm{dL})$ & 475 & 446 & c & c \\
\hline D-dimer $(\mu \mathrm{g} / \mathrm{mL})$ & $3.47^{\mathrm{a}}$ & $3.75^{a}$ & $3.87^{\mathrm{a}}$ & $3.23^{a}$ \\
\hline Troponin-I (ng/mL) & $<0.015$ & $<0.015$ & c & c \\
\hline
\end{tabular}

a , high.

b, low.

', not obtained.

decompensation in the post-partum period for two patients who presented with asymptomatic COVID-19 infection. ${ }^{12}$

In general, with most serious maternal conditions, the goal of therapy is to provide supportive care and treatment to stabilize the mother with the intent of avoiding delivery during the time of systemic infection or inflammation. This is generally done while monitoring the fetus, reserving delivery for obstetric indications including a nonreassuring fetal status. In providing care for our critically ill pregnant woman with severe COVID-19 pneumonia, the team was faced with additional challenges such as fetal monitoring, the logistics of an emergency cesarean delivery, and the risk of COVID-19 exposure to the health care team in the case of an obstetric emergency.

Our multidisciplinary team held several bedside meetings to discuss management and the merit of delivery. The argument against planned delivery was associated with the fact that the stress and increased oxygen requirements of labor or cesarean delivery would result in a need for intubation and subsequent difficulty with extubation given her active lung infection. The intensivists strongly advocated for expectant management with close monitoring; we agreed to delay delivery unless intubation was required for another reason.

Data on ideal timing of delivery in the setting of severe COVID-19 infection are limited. ${ }^{2,4}$ Our case shows that delaying delivery is a reasonable option, even with severe disease.
Furthermore, prognostic laboratory parameters may be helpful (severe lymphopenia, elevated D-dimer, interleukin-6, and ferritin levels) to determine the risk of worse outcomes. ${ }^{13-15}$ Our patient's only concerning laboratory findings were lymphopenia and an elevated D-dimer though D-dimer levels increase in normal pregnancy. ${ }^{16}$ No other laboratory abnormalities consistent with worsening decompensation were seen.

Strikingly, our patient developed cholecystitis after recovering from the pulmonary aspects of COVID-19, an unusual clinical condition in a 16-year-old without obesity or a significant medical history. Gallbladder pathology is not commonly seen during pregnancy, reported as 0.05 to $0.3 \% .{ }^{17-19}$ COVID-19 uses the ACE-2 receptor to gain entry into cells. ${ }^{3}$ This receptor is expressed in epithelial cells of the lung, kidney, heart, and multiple digestive tract organs including the liver and gallbladder. ${ }^{5}$ We suspect that the gallbladder can be a site for COVID-19 infection since this receptor has been identified in the gallbladder. Though there have been reports of liver injury and dysfunction with COVID-19, to our knowledge this is the first case of gallbladder pathology, particularly cholecystitis, related to the virus.

\section{Conclusion}

This case illustrates several important points: first, close collaboration of a multidisciplinary team is paramount in the 
management of pregnant women with severe manifestations of COVID-19. Second, expectant management and delaying delivery should be considered, even in patients with severe disease. Finally, we demonstrate the possibility that inflammation of the gallbladder may be due to underlying COVID-19 infection.

\section{Conflict of Interest}

The authors report no conflict of interest.

\section{References}

1 LoMauro A, Aliverti A. Respiratory physiology of pregnancy: physiology masterclass. Breathe (Sheff) 2015;11(04):297-301

2 Chen D, Yang H, Cao Y, et al. Expert consensus for managing pregnant women and neonates born to mothers with suspected or confirmed novel coronavirus (COVID-19) infection. Int J Gynaecol Obstet 2020;149(02):130-136

3 Tolcher MC, McKinney JR, Eppes CS, et al. Prone positioning for pregnant women with hypoxemia due to coronavirus disease 2019 (COVID-19). Obstet Gynecol 2020;136(02):259-261

4 Halscott T, Vaught J. Society for Maternal Fetal Medicine Management Considerations for Pregnant Patients with COVID-19. Clinical Guidance April 30. Available at: https://s3.amazonaws.com/ cdn.smfm.org/media/2336/SMFM_COVID_Management_of_COVID_pos_preg_patients_4-30-20_final.pdf. July 30, 2020

5 Bavishi C, Maddox TM, Messerli FH. Coronavirus disease 2019 (COVID-19) infection and renin angiotensin system blockers. JAMA Cardiol 2020;5(07):745-747

6 Juusela A, Nazir M, Gimovsky M. Two cases of coronavirus 2019related cardiomyopathy in pregnancy. Am J Obstetrics Gynecology MFM 2020;2(02):100113

7 Zhang C, Shi L, Wang F-S. Liver injury in COVID-19: management and challenges. Lancet Gastroenterol Hepatol 2020;5(05): 428-430

8 Cheng Y, Luo R, Wang K, Zhang M, Wang Z, Dong L, Li J, Yao Y, Ge S, Xu G. Kidney disease is associated with in-hospital death of patients with COVID-19. Kidney Int 2020;97(05):829-838

9 Abbott Press Release Abbott releases interim clinical study data on ID NOW COVID-19 rapid test showing strong agreement to labbased molecular PCR tests, May 21, 2020. https://abbott.medi- calaroom.com/2020-05-21-abbott-release-interim-clinicalstudy-data-an-id-nov-covid19-rapid-test-showing-strongagreement-to-lab-base-molecular-pcr-test. Accessed July 5, 2020

10 Basu A, Zinger T, Inglima K, et al. Performance of Abbott ID Now COVID-19 rapid nucleic acid amplification test using nasopharyngeal swabs transported in viral transport media and dry nasal swabs in a New York City academic institution. J Clin Microbiol 2020;58(08):e01136-e20

11 Spong CY, Mercer BM, D’alton M, Kilpatrick S, Blackwell S, Saade G. Timing of indicated late-preterm and early-term birth. Obstet Gynecol 2011;118(2 Pt 1):323-333

12 Breslin N, Baptiste C, Gyamfi-Bannerman C, et al. Coronavirus disease 2019 infection among asymptomatic and symptomatic pregnant women: two weeks of confirmed presentations to an affiliated pair of New York City hospitals. Am J Obstet Gynecol MFM 2020;2(02):100118

13 Zhou F, Yu T, Du R, et al. Clinical course and risk factors for mortality of adult inpatients with COVID-19 in Wuhan, China: a retrospective cohort study. Lancet 2020;395(10229):1054-1062

14 Henry BM, de Oliveira MHS, Benoit S, Plebani M, Lippi G. Hematologic, biochemical and immune biomarker abnormalities associated with severe illness and mortality in coronavirus disease 2019 (COVID-19): a meta-analysis. Clin Chem Lab Med 2020;58 (07):1021-1028

15 Pierce-Williams R, Burd J, Felder L, et al. Clinical course of severe and critical coronavirus disease 2019 in hospitalized pregnancies: a United States cohort study. Am J Obstet Gynecol MFM 2020;2 (03):100134

16 Kline JA, Williams GW, Hernandez-Nino J. D-dimer concentrations in normal pregnancy: new diagnostic thresholds are needed. Clin Chem 2005;51(05):825-829

17 Ellington SR, Flowers L, Legardy-Williams JK, Jamieson DJ, Kourtis AP. Recent trends in hepatic diseases during pregnancy in the United States, 2002-2010. Am J Obstet Gynecol 2015;212(04): 524.e1-524.e7

18 Date RS, Kaushal M, Ramesh A. A review of the management of gallstone disease and its complications in pregnancy. Am J Surg 2008;196(04):599-608

19 Elamin Ali M, Yahia Al-Shehri M, Abu-Eshy S, Cheema MA, Mustafa Z, Sadek A. Is surgical intervention in acute cholecystitis in pregnancy justified? J Obstet Gynaecol 1997;17(05):435-438 